\title{
Etude expérimentale des effets biologiques d'une irradiation gamma pendant les périodes intra-utérine et post-natale chez le rat
}

\author{
Michèle MORIN, Jeanine BONCORPS et Roland MASSE* \\ (Manuscrit reçu le 18 août 1988)
}

\begin{abstract}
RÉSUMÉ
Cette étude a été entreprise pour connaitre les effets carcinogénétiques d'une irradiation globale sur des rats exposés pendant les périodes utérine et postnatale et les comparer aux effets obtenus après irradiation de jeunes adultes. 8 jours après accouplement, des rates Sprague-Dawley sont irradiées 5 heures par jour et 5 jours par semaine par une source de cobalt 60 ; leurs portées sont retirées à des temps variables à partir du jour de la naissance et suivies jusqu'à leur mort spontanée. D'une part, l'irradiation in utero provoque des diminutions sensibles de la croissance du cerveau et des gonades; d'autre part, les rats irradiés pendant la période périnatale, à des doses de 2,66, 5,96 et 14,74 Gy, montrent une sensibilité élevée à l'induction de tumeurs du cerveau et des gonades, mais aucune sensibilité particulière à l'induction des tumeurs du système hématopoïétique.
\end{abstract}

\section{ABSTRACT}

This study was carried out to examine the effects of protracted gamma irradiation in rats irradiated during development in comparison to rats irradiated at the young adult period. Sprague-Dawley pregnant rats were exposed 5 hours per day to whole-body irradiation from a cobalt 60 source from 8 days post-coìtus, and the litters were removed at different times after parturition. In utero irradiation of rats produced significant changes in growth and development of brain and gonads. Perinatally-irradiated rats $(2.66,5.96$ and $14.74 \mathrm{~Gy})$ showed higher susceptibility to induction of brain and gonad tumors and no increased susceptibility to induction of hematopoïetic tumors (lymphomas and myeloïd leukaemias).

* Commissariat à l'énergie atomique, IPSN, Département de protection sanitaire, BP 6 , 92265 Fontenay-aux-Roses Cedex. 


\section{INTRODUCTION}

L'âge au moment de l'irradiation est un facteur important en radiocancérogenèse. Les effets des rayonnements sont-ils les mêmes selon les stades du développement de l'organisme au moment de l'irradiation ?

Les normes de radioprotection ont été établies pour l'adulte, mais la sensibilité d'un organisme est peut-être différente pendant la période foetale. C'est ce qu'ont semblé démontrer certaines études faites sur la souris et sur le chien [1-4].

Cette étude a été faite pour comparer les effets, sur la durée de vie et l'apparition des cancers, d'une irradiation périnatale aux effets d'une irradiation chez le jeune adulte.

\section{MATÉRIEL ET MÉTHODES}

Des rats de souche Sprague-Dawley SPF ont été utilisés pour cette étude; 8 jours après accouplement, 30 femelles ont été irradiées par les rayonnements gamma d'une source de cobalt 60 à un débit de 5,3 cGy par heure, $5 \mathrm{~h}$ par jour, 5 jours par semaine.

- Série 1: après 2 semaines d'irradiation globale, au moment de la mise bas, 10 femelles et leurs portées (65 femelles et 66 mâles) sont retirées de l'irradiation après avoir reçu une dose moyenne de 266 cGy.

- Série 2: les 20 autres femelles et leurs portées restent en irradiation, mais seulement $3 \mathrm{~h}$ par jour pendant encore 1 mois, jusqu'au sevrage des nouveau-nés. On enlève alors toutes les mères et une partie des portées, soit 63 femelles et 69 mâles. Ces animaux ont reçu au total en moyenne 596 cGy.

- Série 3: on laisse en irradiation 56 femelles et 48 mâles, qui sont séparés par sexe et irradiés $3 \mathrm{~h}$ par jour, 5 jours par semaine pendant encore 16 semaines. Cette dernière série a reçu en moyenne 1474 cGy.

Une des femelles n'était pas fécondée. La moyenne par portée chez les mères irradiées était de 12,7 nouveau-nés vivants, 2 mères témoins ont eu, pendant le même temps, 16 et 17 nouveau-nés. Nous avions au total 400 nouveau-nés à suivre jusqu'à leur mort spontanée.

\section{RÉSULTATS}

\section{Action sur la durée de vie}

Pour l'ensemble des deux premières séries, 3,5\% des nouveau-nés sont morts avant 400 jours; dans la dernière série, $15 \%$ des nouveau-nés meurent avant cette date. Chez les témoins, on observe $3 \%$ de morts avant 400 jours. II n'y a donc pas de phénomène détectable de mortalité précoce; 
de même, chez les nouveau-nés irradiés, aucune malformation n'a été observée à l'autopsie de routine. Un raccourcissement de la durée de vie en fonction de la dose est observé (tableau I).

TABLEAU I

Action de l'irradiation néonatale sur la durée de vie

\begin{tabular}{|c|c|c|c|c|c|}
\hline $\begin{array}{l}\text { Période de développement } \\
\text { au moment de l'irradiation }\end{array}$ & $\begin{array}{l}\text { Dose } \\
\text { (cGy) }\end{array}$ & $\begin{array}{c}\text { Nombre } \\
? \\
q\end{array}$ & de rats & $\begin{array}{l}\text { Durée de } \\
\wp\end{array}$ & $\begin{array}{c}\text { vie (jours) } \\
\sigma^{\prime}\end{array}$ \\
\hline Témoins & 0 & 20 & 13 & $\begin{array}{c}771 \\
(396 \text { à } 960)\end{array}$ & $\begin{array}{c}803 \\
(629 \text { à } 960)\end{array}$ \\
\hline $\begin{array}{l}\text { Série } 1=\text { fœtus } \\
8 \text { jpc à parturition }\end{array}$ & 266 & 65 & 66 & $\left(449^{765}\right.$ à 958$)$ & $\begin{array}{c}737 \\
(165 \text { à } 958)\end{array}$ \\
\hline $\begin{array}{l}\text { Série } 2=\text { fœtus et } \\
\text { nouveau-nés avant sevrage } \\
8 \text { jpc à } 30 \text { jpp }\end{array}$ & 596 & 63 & 69 & $\begin{array}{c}674 \\
\left(113^{\text {à }} 958\right)\end{array}$ & $\left(344^{708}\right.$ à 917$)$ \\
\hline $\begin{array}{l}\text { Série } 3=\text { fœtus, } \\
\text { nouveau-nés, } \\
\text { jeunes post-sevrage } \\
8 \text { jpc à } 140 \text { jpp }\end{array}$ & 1474 & 56 & 48 & $\begin{array}{c}555 \\
(186 \text { à } 958)\end{array}$ & $(239 \stackrel{582}{\text { à }} 864)$ \\
\hline $\begin{array}{l}\text { (pour comparaison) }= \\
\text { jeunes adultes } \\
90 \text { jpp }\end{array}$ & 300 & & 120 & & (138 à 996) \\
\hline
\end{tabular}

\section{2. Étude pondérale des nouveau-nés irradiés}

Les animaux sont pesés 1 mois après leur naissance, puis à 270 jours, 1 an et 568 jours. Les organes sont pesés au moment de l'autopsie avant fixation dans du Bouin-Hollande.

- Poids total: seuls les animaux irradiés à 1474 cGy présentent une diminution de poids. Cette diminution, de l'ordre de $15 \%$ par rapport aux témoins, reste constante au cours du temps (fig. 1A-1C).

1A. Poids des femelles

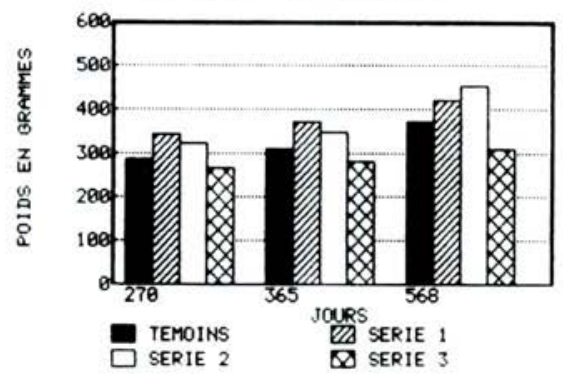

1B. Poids des mâles

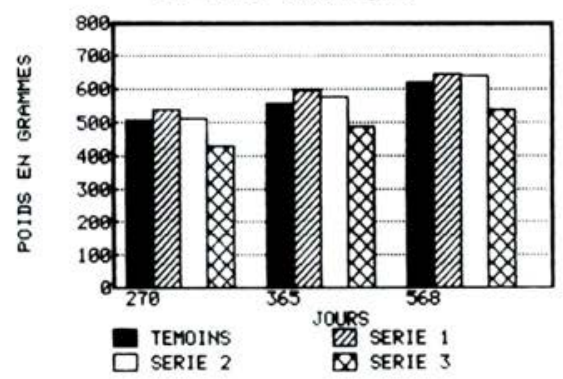

Fig. 1A et 1 B. - Evolution pondérale moyenne des portées femelles (1A) et des portées mâles (1B). 


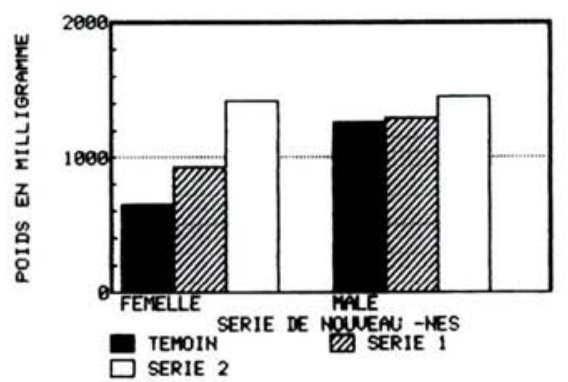

Fig. 1C. - Comparaison à l'âge de 1 mois du poids des portées mâles et femelles chez les animaux témoins et irradiés.

- Poids des reins, du foie et de la rate: chez les femelles irradiées le poids des reins et du foie est équivalent à celui des témoins. Chez les mâles irradiés on constate une perte de poids du foie pour la dose la plus élevée, et une diminution du poids des reins pour les doses moyennes et fortes. Le poids de la rate des animaux irradiés, pour les deux sexes et pour toutes les doses, est toujours légèrement supérieur à celui de la rate des animaux témoins. L'irradiation entraîne une érythropoïèse entretenue dans les sinus spléniques et une augmentation du volume de la rate (fig. 2A-2C).

2A. Poids des reins

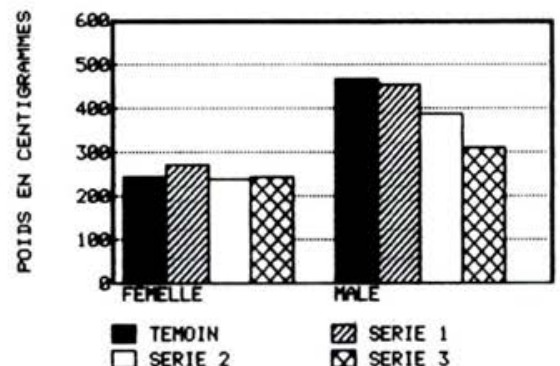

2B. Poids du foie

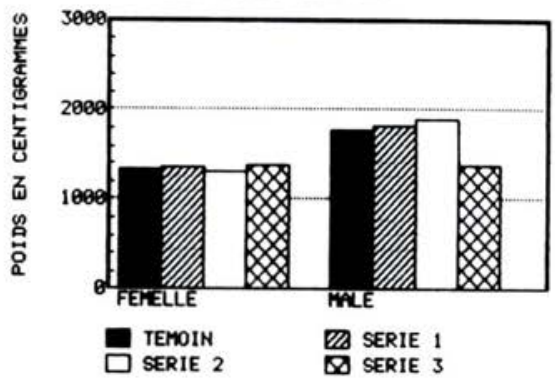

2C. Poids de la rate

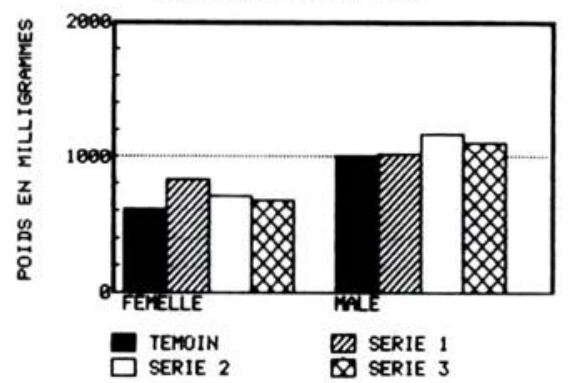

Fig. 2A - 2C. - Poids des reins, du foie et de la rate dans les différentes séries d'animaux au moment de la mort. 
- Poids du cerveau: alors que l'irradiation chez l'adulte ne modifie pas le poids du cerveau, l'irradiation du foetus diminue le poids de cet organe. La diminution de poids est sensiblement la même dans les deux premières séries, la perte de poids dans la troisième série est légèrement plus élevée. La radiosensibilité est donc maximale avant la naissance (fig. 3).

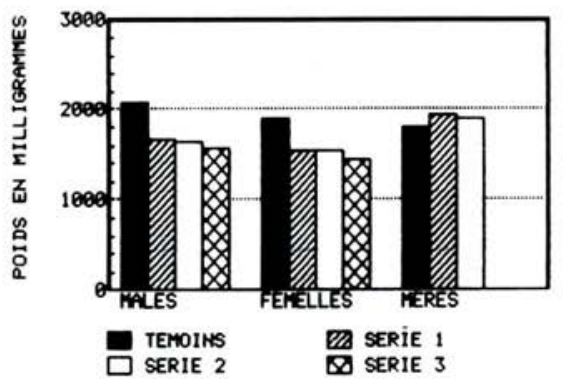

Fig. 3. - Poids du cerveau dans les différentes séries mâles et femelles.

- Poids des testicules: la diminution observée sur le poids des testicules est très importante. La radiosensibilité maximale est située avant la naissance, puisqu'une perte de $82 \%$ du poids de cet organe apparaît déjà dans la première série. Une irradiation postnatale amplifie le phénomène, mais dans tous les cas, les testicules sont atrophiés et stériles (fig. 4).

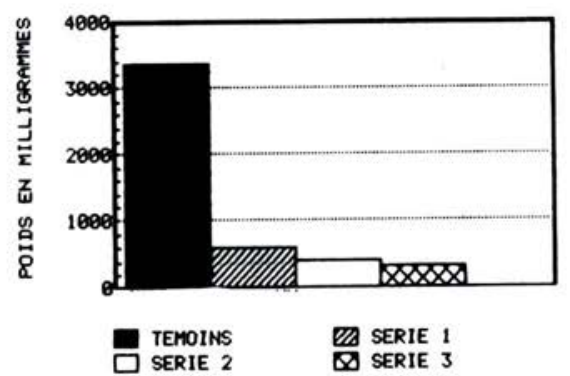

Fig. 4. - Poids des testicules dans les différentes séries de mâles.

Les ovaires n'ont pas été systématiquement pesés, mais ils sont toujours atrophiés. 


\section{Induction de tumeurs}

\section{- Tumeurs malignes (fig. 5) :}

Le pourcentage élevé de cancers observés chez les témoins mâles est statistiquement peu représentatif étant donné le trop faible nombre d'animaux: 13 rats. Le suivi que nous avons fait pour d'autres expériences sur 600 mâles témoins n'a fait apparaître que $31 \%$ de cancers au lieu des $69 \%$ trouvés ici. Une série de 120 rats mâles irradiés à l'âge de 3 mois à la dose de 300 cGy nous sert de point de comparaison pour la première série de mâles irradiés à 266 cGy. Les femelles des deux premières séries sont respectivement comparées aux mères irradiées pendant le même temps.

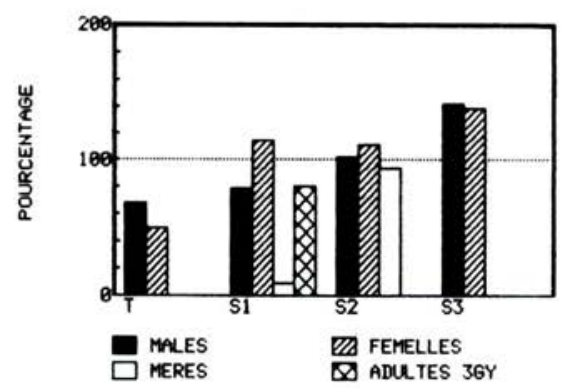

Fig. 5. - Pourcentages de cancers observés

dans les séries irradiées en période périnatale;

comparaison avec des animaux irradiés à l'âge adulte.

En ce qui concerne l'induction de cancers, deux systèmes d'organes apparaissent comme particulièrement sensibles pendant l'organogenèse chez le rat: le système nerveux central et les gonades.

Dès 266 cGy, la fréquence de cancers du cerveau double chez les animaux irradiés pendant la période fœtale, alors que pour une dose équivalente délivrée chez de jeunes adultes, cette fréquence reste égale à celle des témoins (fig. 6).

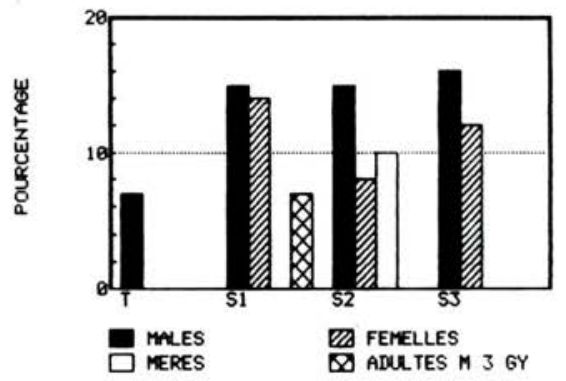

Fig. 6. - Pourcentages de cancers du cerveau dans les séries irradiées en période périnatale; comparaison avec des adultes irradiés. 
L'augmentation importante de cancers chez les femelles irradiées en période périnatale est principalement due au pourcentage élevé de cancers du stroma ovarien, Au contraire, les mères irradiées n'ont présenté aucun cancer de l'ovaire (fig. 7).

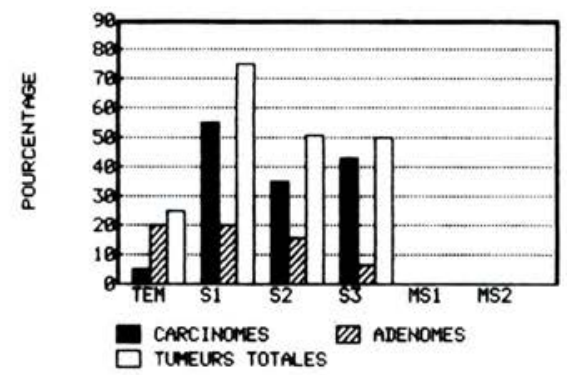

Fig. 7. - Pourcentages de tumeurs de l'ovaire; comparaison entre irradiation périnatale et irradiation chez l'adulte.

Le nombre de tumeurs mammaires est, pour une même dose, 2 fois moins élevé chez les femelles irradiées à l'état fœtal que chez les mères irradiées (fig. 8).

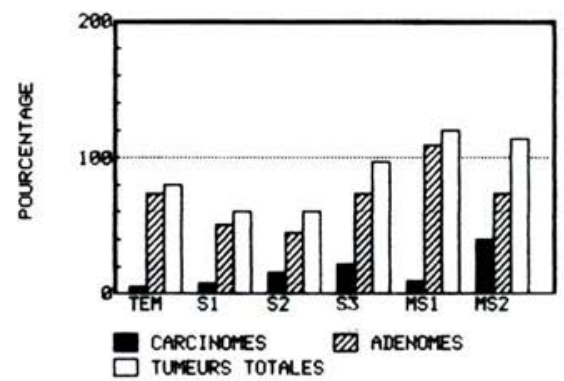

Fig. 8. - Pourcentages de tumeurs mammaires; comparaison entre irradiation périnatale et irradiation chez l'adulte.

La fréquence des tumeurs de l'hypophyse n'est pas sensiblement augmentée, mais leur développement semble plus intense et entraîne souvent un envahissement du cerveau plus important que chez l'adulte irradié.

Les tumeurs de la surrénale sont un peu plus fréquentes que chez l'adulte irradié, en particulier chez les mâles.

L'incidence des cancers pulmonaires après irradiation est plus élevée chez les mâles que chez les femelles, mais elle n'est pas plus élevée chez les rats irradiés pendant la période périnatale que chez les adultes irradiés à la même dose. 
Enfin, aucun excès des autres cancers n'a été constaté par rapport aux adultes irradiés et, en particulier aucune augmentation de lymphosarcome ni de leucémie n'a été observée (tableaux II - IV).

TABLEAU ॥

Tumeurs malignes des femelles

(\% du nombre total d'animaux par série)

\begin{tabular}{|c|c|c|c|c|c|c|}
\hline & $\underset{(20)}{\text { Témoins }}$ & $\underset{(65)}{S}$ & $\underset{(63)}{S}$ & $\begin{array}{c}\text { Série } 3 \\
(56)\end{array}$ & $\begin{array}{c}\text { Mères série } 1 \\
(10)\end{array}$ & $\begin{array}{c}\text { Mères série } 2 \\
(20)\end{array}$ \\
\hline Ovaires & 5 & 56 & 35 & 43 & 0 & 0 \\
\hline $\begin{array}{l}\text { Glandes } \\
\text { mammaires }\end{array}$ & 5 & 9 & 16 & 23 & 10 & 40 \\
\hline Cerveau & 0 & 14 & 8 & 13 & 0 & 10 \\
\hline Hypophyse & 15 & 15 & 16 & 5 & 0 & 0 \\
\hline Thyroïde & 5 & 4 & 1 & 0 & 0 & 0 \\
\hline Surrénales & 10 & 0 & 10 & 3 & 0 & 5 \\
\hline $\begin{array}{l}\text { Leucémie- } \\
\text { lymphosarcome }\end{array}$ & 0 & 0 & 3 & 7 & 0 & 5 \\
\hline Autres & 10 & 16 & 22 & 45 & 0 & 35 \\
\hline TOTAL & 50 & 114 & 111 & 139 & 10 & 95 \\
\hline
\end{tabular}

TABLEAU III

Tumeurs malignes des mâles

(\% du nombre total d'animaux par série)

\begin{tabular}{|l|c|r|r|r|c|}
\hline & $\begin{array}{c}\text { Témoins } \\
(13)\end{array}$ & $\begin{array}{c}\text { Série 1 } \\
(66)\end{array}$ & $\begin{array}{c}\text { Série 2 } \\
(69)\end{array}$ & $\begin{array}{c}\text { Série 3 } \\
(48)\end{array}$ & $\begin{array}{c}\text { Jeunes adultes 3 Gy } \\
(120)\end{array}$ \\
\cline { 2 - 6 } Testicules & 0 & 6 & 20 & 8 & 0 \\
Cerveau & 7 & 15 & 15 & 17 & 7 \\
Hypophyse & 16 & 3 & 11 & 8 & 3 \\
Thyroïde & 7 & 6 & 2 & 2 & 17 \\
Surrénales & 0 & 13 & 10 & 2 & 6 \\
Leucémie- & 0 & 1 & 1 & 13 & 3 \\
lymphosarcome & 39 & 34 & 42 & 92 & 45 \\
Autres & 69 & 79 & 101 & 142 & 81 \\
\hline TOTAL & & & & & \\
\hline
\end{tabular}


TABLEAU IV

Tumeurs malignes de quelques organes du système

(\% du nombre total d'animaux par série)

\begin{tabular}{|l|r|r|r|r|r|r|r|}
\hline & Reins & Os & Pancréas & Poumons & $\begin{array}{c}\text { Système } \\
\text { vasculaire }\end{array}$ & Peau & Foie \\
\cline { 2 - 7 } & & & & & & & \\
FEMELLES & & & & & & & \\
20 témoins & 0 & 0 & 0 & 0 & 0 & 0 & 5 \\
65 série 1 & 0 & 0 & 1 & 0 & 3 & 1 & 1 \\
63 série 2 & 3 & 2 & 0 & 0 & 3 & 5 & 1 \\
56 série 3 & 6 & 0 & 0 & 7 & 11 & 3 & 0 \\
10 mères série 1 & 0 & 0 & 0 & 0 & 0 & 0 & 0 \\
20 mères série 2 & 5 & 0 & 0 & 0 & 0 & 5 & 0 \\
& & & & & & & \\
MÂLES : & & & & & & \\
13 témoins & 8 & 0 & 0 & 0 & 0 & 0 & 7 \\
66 série 1 & 2 & 0 & 3 & 7 & 2 & 0 & 1 \\
69 série 2 & 7 & 2 & 8 & 2 & 6 & 3 & 3 \\
48 série 3 & 13 & 4 & 10 & 9 & 16 & 16 & 0 \\
120 jeunes adultes & 8 & 2 & 3 & 7 & 3 & 6 & 3 \\
(3 Gy) & & & & & & & \\
\hline
\end{tabular}

- Métastases (tableau V):

Sur 455 cancers détectés, 61 ont métastasé, soit $13,4 \%$; certains cancers métastasent dans plusieurs organes. Les tumeurs qui métastasent le plus sont les cancers de l'ovaire, les sarcomes des tissus mous, et les carcinomes des glandes mammaires. Les métastases interviennent surtout dans le poumon, mais de nombreuses métastases sont aussi trouvées dans le cerveau - 5 des 14 cancers de l'ovaire et 5 des 6 cancers de l'hypophyse ont des métastases dans le cerveau. Aucune différence due à l'âge n'a été observée.

\section{- Tumeurs bénignes (fig. 9A):}

Le pourcentage de tumeurs bénignes est plus élevé chez les femelles que chez les mâles, mais ceci est dû au nombre élevé de tumeurs de l'ovaire et des glandes mammaires. Ce phénomène disparaît et même s'inverse, si l'on élimine les tumeurs génitales. Ce fait se retrouve chez les adultes irradiés (fig. 9B, tableaux VI - VII). 


\section{TABLEAU V}

Métastases

\begin{tabular}{|c|c|c|}
\hline Cancers métastasant & Nombre de métastases & Organes métastasés \\
\hline - adenocarc. ovaires & $\begin{array}{l}6 \\
5 \\
3 \\
1 \\
1\end{array}$ & $\begin{array}{l}\text { poumon } \\
\text { cerveau } \\
\text { ggl. pulm. et médiastin } \\
\text { rein } \\
\text { surrénale }\end{array}$ \\
\hline $\begin{array}{l}\text { - sarc. tissus mous } \\
\text { (surtout RMS) }\end{array}$ & $\begin{array}{l}7 \\
3 \\
2 \\
1 \\
1 \\
1\end{array}$ & $\begin{array}{l}\text { poumon } \\
\text { rate } \\
\text { poumons et ggl. pulmonaires } \\
\text { rein } \\
\text { surrénale } \\
\text { foie }\end{array}$ \\
\hline - adenocarc. mammaire & $\begin{array}{l}6 \\
2 \\
1 \\
1\end{array}$ & $\begin{array}{l}\text { poumon } \\
\text { ggl. pulmonaires } \\
\text { ggl. axillaires } \\
\text { mâchoire }\end{array}$ \\
\hline - mésenchym. mammaire & $\begin{array}{l}1 \\
1\end{array}$ & $\begin{array}{l}\text { plèvre et médiastin } \\
\text { tissus mous }\end{array}$ \\
\hline - cancer hypophyse & $\begin{array}{l}5 \\
1 \\
1\end{array}$ & $\begin{array}{l}\text { cerveau } \\
\text { ggl. pulmonaires } \\
\text { surrenale }\end{array}$ \\
\hline - ostéosarcome & $\begin{array}{l}3 \\
1 \\
1\end{array}$ & $\begin{array}{l}\text { poumon } \\
\text { rein } \\
\text { foie }\end{array}$ \\
\hline - carc. thyroïde & $\begin{array}{l}1 \\
1 \\
1\end{array}$ & $\begin{array}{l}\text { poumon } \\
\text { ggl. pulmonaires } \\
\text { surrenale }\end{array}$ \\
\hline - adenocarc. pancréas & $\begin{array}{l}1 \\
1\end{array}$ & $\begin{array}{l}\text { ggl. pulmonaires } \\
\text { ch. lymph. thorac. }\end{array}$ \\
\hline $\begin{array}{l}\text { - adenocarc. rein } \\
\text { - mésenchym. rein }\end{array}$ & $\begin{array}{l}1 \\
1\end{array}$ & $\begin{array}{l}\text { foie } \\
\text { poumon }\end{array}$ \\
\hline - hépatocarcinome & $\begin{array}{l}1 \\
1\end{array}$ & $\begin{array}{l}\text { cerveau } \\
\text { plèvre et médiastin }\end{array}$ \\
\hline - angiosarcome & $\begin{array}{l}3 \\
2\end{array}$ & $\begin{array}{l}\text { poumon } \\
\text { ggl. pulm. et médiastin }\end{array}$ \\
\hline $\begin{array}{l}\text { - carcinome testicule } \\
\text { (cell. de Leydig) }\end{array}$ & $\begin{array}{l}1 \\
1\end{array}$ & $\begin{array}{l}\text { poumon } \\
\text { autre testicule }\end{array}$ \\
\hline - carc. gl. salivaires & 2 & poumon \\
\hline $\begin{array}{l}\text { - corticosurrénalome } \\
\text { - phéochromocytome }\end{array}$ & $\begin{array}{l}1 \\
1\end{array}$ & $\begin{array}{l}\text { foie } \\
\text { poumon et ggl. pulmonaires }\end{array}$ \\
\hline - carc. vessie & 1 & cerveau \\
\hline - léiomyosarc. estomac & 1 & poumon \\
\hline - cancer larynx & 1 & poumon \\
\hline - carc. épiderm. kératinisé & 1 & cerveau \\
\hline - inconnu & 1 & poumon \\
\hline
\end{tabular}


EFFETS BIOLOGIQUES DE L'IRRADIATION GAMMA PENDANT LA PÉRIODE PERI-NATALE

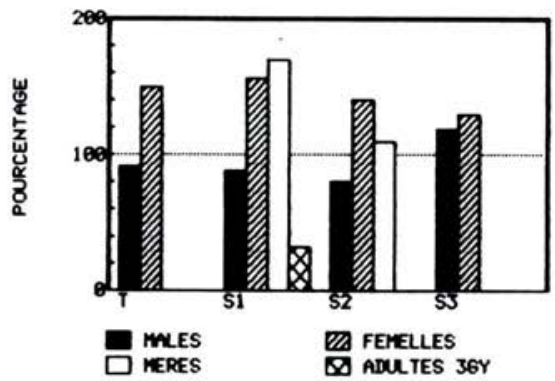

Fig. 9A. - Pourcentages de tumeurs bénignes observées dans les séries irradiées en période périnatale; comparaison avec des animaux irradiés à l'âge adulte.

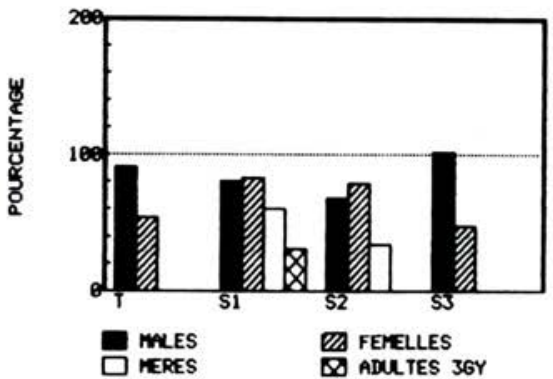

Fig. 9B. - Pourcentages de tumeurs bénignes observées après avoir ôté les tumeurs génitales et mammaires.

TABLEAU VI

Tumeurs bénignes des femelles

(\% du nombre total d'animaux par série)

\begin{tabular}{|l|r|r|r|r|r|r|}
\hline & $\begin{array}{c}\text { Témoins } \\
(20)\end{array}$ & $\begin{array}{c}\text { Série } \\
(65)\end{array}$ & $\begin{array}{c}\text { Série } 2 \\
(63)\end{array}$ & $\begin{array}{c}\text { Série 3 } \\
(56)\end{array}$ & $\begin{array}{c}\text { Mères série } 1 \\
(10)\end{array}$ & $\begin{array}{c}\text { Mères série 2 } \\
(20)\end{array}$ \\
\cline { 2 - 7 } Ovaires & 20 & 20 & 16 & 7 & 0 & 0 \\
Glandes & 75 & 52 & 45 & 75 & 110 & 75 \\
mammaires & & & & & & 0 \\
Cerveau & 0 & 5 & 8 & 3 & 0 & 15 \\
Hypophyse & 30 & 37 & 33 & 9 & 20 & 5 \\
Thyroïde & 15 & 11 & 10 & 6 & 0 & 0 \\
Surrénales & 0 & 9 & 9 & 7 & 0 & 15 \\
Autres & 10 & 22 & 19 & 23 & 40 & 110 \\
\cline { 2 - 7 } TOTAL & 150 & 156 & 140 & 130 & 170 & \\
\hline
\end{tabular}


TABLEAU VII

Tumeurs bénignes des mâles

(\% du nombre total d'animaux par série)

\begin{tabular}{|l|c|c|c|c|c|}
\hline & $\begin{array}{c}\text { Témoins } \\
(13)\end{array}$ & $\begin{array}{c}\text { Série } 1 \\
(66)\end{array}$ & $\begin{array}{c}\text { Série } 2 \\
(69)\end{array}$ & $\begin{array}{c}\text { Série 3 } \\
(48)\end{array}$ & $\begin{array}{c}\text { Jeunes adultes 3 Gy } \\
(120)\end{array}$ \\
\cline { 2 - 6 } Testicules & 0 & 9 & 11 & 16 & 0 \\
Cerveau & 7 & 3 & 6 & 10 & 0 \\
Hypophyse & 23 & 21 & 13 & 13 & 5 \\
Thyroïde & 39 & 21 & 5 & 9 & 6 \\
Surrénales & 0 & 15 & 9 & 17 & 0 \\
Autres & 23 & 20 & 36 & 54 & 22 \\
\cline { 2 - 6 } TOTAL & 92 & 89 & 80 & 119 & 33 \\
\hline
\end{tabular}

\section{CONCLUSION}

La comparaison des effets d'une même dose d'irradiation gamma globale délivrée sur des rats en période périnatale ou sur des rats adultes montre une radiosensibilité élevée du cerveau et des gonades chez les fœtus pendant la période de formation.

Cette sensibilité constatée chez les rats irradiés pendant leur période d'organogenèse se traduit, d'une part, par une diminution du développement du cerveau et des gonades et, d'autre part, par une incidence des tumeurs de ces organes plus élevée chez les rats irradiés en période fœtale que chez les rats adultes.

On n'a pas constaté d'excès de leucémies ou de lymphosarcomes, mais il existe un déficit de tumeurs mammaires chez les fœtus irradiés. Le déficit des tumeurs mammaires peut être interprété comme une conséquence de l'atrésie ovarienne, compte tenu des interactions connues entre hormones œstrogènes et cancérogenèse mammaire [2].

L'augmentation des tumeurs nerveuses et de l'ovaire après irradiation pendant la période fœtale indique que tous les stades de différenciation cellulaire n'ont pas la même susceptibilité. Ce caractère pourrait être dû à un plus grand nombre relatif de cellules aptes à la division cellulaire; cependant, il est remarquable que le phénomène ne soit pas généralisable à l'ensemble des organes. II est donc vraisemblable qu'il s'agisse d'un phénomène liè à l'état de différenciation cellulaire. 


\section{RÉFÉRENCES}

[1] BENJAMIN S.A., THOMASSEN R.W., ANGLETON G.M. et al. - Effect of whole-body irradiation during prenatal and early postnatal development in the beagle dog. Radiat. Res., 1978, 74, 514.

[2] BLANKENSTEIN M.A., BROERSE J.J., DE VRIES J.B., et al. - The effect of subcutaneous administration on plasma oestrogen levels and tumour incidence in female rats. Eur. J. Cancer, 1977, 13, 1437.

[3] PHEMISTER R.D., THOMASSEN R.W., ANGLETON G.M. et al. Early malignant neoplasms in dogs irradiated during the prenatal period. Radiat. Res., 1977, 70, 669-670.

[4] SASAKI S., and KASUGA T. - Life-shortening and carcinogenesis in mice irradiated at the perinatal period with gamma rays. In: Life-span radiation effects studies in animals: what can they tell us ? 22 Hanford life science symposium, Richland, Washington, 27-29 Sept. 1983 (CONF- 830951). Springfield: NTIS, 1986, 357-367. 